\title{
Observational Equivalence of Deterministic and Indeterministic Descriptions and the Role of Different Observations
}

\author{
Charlotte Werndl \\ Department of Philosophy, Logic and Scientific Method, London School of Economics and \\ Political Science, Houghton Street, London WC2A 2AE, England \\ c.s.wernd1@1se.ac.uk
}

\section{Introduction}

Recently some results have been presented which show that certain kinds of deterministic descriptions and indeterministic descriptions are observationally equivalent (Werndl 2009a, Werndl 2011). These results prompt interesting philosophical questions, such as what exactly they show or whether the deterministic or indeterministic description is preferable. There is hardly any philosophical discussion about these questions, and this paper contributes to filling this gap.

More specifically, first, I discuss the philosophical comments made by mathematicians about observational equivalence, in particular Ornstein and Weiss (1991). Their comments are vague, and I argue that, according to a reasonable interpretation, they are misguided. Second, the results on observational equivalence raise the question of whether the deterministic or indeterministic description is preferable relative to evidence. If none of them is preferable, there is underdetermination. I criticise Winnie's (1998) argument that, by appealing to different observations, one finds that the deterministic description is preferable. In particular, I clarify a confusion in this argument. Furthermore, I argue that if the concern is a strong form of underdetermination, the argument delivers the desired conclusion but this conclusion follows from a much simpler argument; and for other kinds of underdetermination the argument fails.

This paper proceeds as follows. Section 1 introduces deterministic and indeterministic descriptions. Section 2 presents the relevant results on observational equivalence. These results are technical, and I will keep the discussion at an intuitive level. Section 3 discusses the mathematicians' claims about observational equivalence. Section 4 is about Winnie's argument on the role of different observations. Section 5 summarises the results. 


\section{$\underline{\text { II. Deterministic and Indeterministic Descriptions }}$}

I now introduce the relevant deterministic and indeterministic descriptions informally; for the technical details see Werndl (2011). There are two kinds of descriptions: either the time-parameter varies discretely or continually. Because the latter case is more important in the sciences, this paper focuses on descriptions involving a continuous time parameter. ${ }^{1}$

\section{II.a Deterministic Descriptions}

We are concerned with measure-theoretic deterministic descriptions, in short deterministic descriptions. A deterministic description is a triple $\left(X, \mathrm{~T}_{\mathrm{t}} \mathrm{p}\right)$; the set $X$ (the phase space), represents all possible states; $\mathrm{T}_{\mathrm{t}}(x), t \in \mathbf{R}$, are functions (the evolution functions) which tell one that the system in state $x$ evolves to $\mathrm{T}_{\mathrm{t}}(x)$ after $t$ time steps; and $\mathrm{p}$ assigns a probability to regions of $X .^{2}$ Clearly, these descriptions are deterministic according to the standard definition, namely that determinism means that two solutions which agree at one time agree at all future times. A solution represents a possible evolution of the system in the course of time. It is a function $\mathrm{s}_{\mathrm{x}}(t): \mathbf{R} \rightarrow \mathrm{X} ; \mathrm{s}_{\mathrm{x}}(t):=\mathrm{T}_{\mathrm{t}}(x)$ for an arbitrary $x \in X$. Deterministic descriptions thus defined are among the most important descriptions in science, e.g., they include all deterministic descriptions in Newtonian mechanics.

Figure 2.1 Two hard balls in a box

Example 1. Two hard balls in a box. This system consists of two balls moving in a three-dimensional box; the balls interact by elastic collisions and have a finite radius but no rotational motion (cf. Simanyi 1999). Figure 1 shows the hard ball system in a specific state. Mathematically, this system is described by a Newtonian description as follows. $X$ is the set of all possible states, i.e., the set of all vectors consisting of the position and velocity coordinates of the two balls. Thus the specific state of the system shown in Figure 1 is represented by exactly one $x \in X$. The evolution functions tell us that the hard ball system in state $x$ evolves to $\mathrm{T}_{\mathrm{t}}(x)$ after $t$ time steps. For an arbitrary region $A$ in phase space, p assigns the probability $\mathrm{p}(A)$ to the event that the two hard balls are in one of the states represented by $A$.

${ }^{1}$ For discrete-time descriptions, see Werndl (2009a).

${ }^{2}$ There are various interpretations of this probability measure. For instance, according to the time-average interpretation, the probability of $A$ is the long-run average of the fraction of time a solution spends in $A$ (Werndl 2009b). 
And a solution represents a possible evolution of the hard ball system in the course of time.

Finally, when a deterministic system in state $x$ is observed, a value $\Phi(x)$ is observed which is dependent on $x$ (but may be different from it). Thus observations are modeled as observation functions, i.e., functions $\Phi: X \rightarrow X_{\mathrm{O}}$ where $X_{\mathrm{O}}$ represents the set of possible observed values.

\section{$\underline{\text { II.b Stochastic Processes }}$}

The indeterministic descriptions which concern us are stochastic descriptions, which model processes governed by probabilistic laws. A stochastic description $\left\{Z_{t}\right\}$ consists of a family of functions $Z_{t}: \Omega \rightarrow E, t \in \mathbf{R}$. The set $E$, called the outcome space, represents all possible outcomes, and $\mathrm{Z}_{\mathrm{t}}(\omega)$ represents the outcome of the process at time $t$. Furthermore, probability distributions $\mathrm{P}\left(\mathrm{Z}_{\mathrm{t}} \in A\right)$ tells us the probability that the process is in $A$ at time $t$ for any region $A$ of $E$ and any $t \in \mathbf{R}$, and probability distributions $\mathrm{P}\left(\mathrm{Z}_{\mathrm{s}} \in A\right.$ given that $\left.\mathrm{Z}_{\mathrm{t}} \in B\right)$ tell us the probability that the process is in $A$ at time $s$ given that it is in $B$ at time $t$ for arbitrary regions $A, B$ of $E$ and any $t, s \in \mathbf{R}$. A realisation represents a possible evolution of the process in the course of time; it is a function $\mathrm{r}_{\omega}(t): \mathbf{R} \rightarrow E, \mathrm{r}_{\omega}(t):=\mathrm{Z}_{\mathrm{t}}(\omega)$ for an arbitrary $\omega \in \Omega$. (Here one sees that, intuitively, $\omega$ encodes the evolution of the stochastic process.) Stochastic descriptions are usually indeterministic. If the description takes a specific outcome, there are many outcomes that might follow, and a probability distribution measures the likelihood of them. Stochastic descriptions are ubiquitous in the sciences.

Figure 2.2 A realisation of a semi-Markov description

Example 2: semi-Markov descriptions. A semi-Markov description has finitely many possible outcomes $\mathrm{e}_{1}, \ldots, \mathrm{e}_{\mathrm{n}}$. The description takes the outcome $\mathrm{e}_{\mathrm{i}}$ for a time $\mathrm{u}_{\mathrm{i}}$, and which outcome follows $\mathrm{e}_{\mathrm{i}}$ depends only on $\mathrm{e}_{\mathrm{i}}$. Figure 2 shows a realisation of a semi-Markov description with four possible outcomes $\mathrm{e}_{1}, \mathrm{e}_{2}, \mathrm{e}_{3}, \mathrm{e}_{4}$. The probability distributions of the semi-Markov description tell us, for instance, the probability that the description takes a specific outcome at time $t$, such as $\mathrm{P}\left(\mathrm{Z}_{\mathrm{t}}=\mathrm{e}_{3}\right)$, or the probability that an outcome at $t$ is followed by another outcome at $s$, e.g., $\mathrm{P}\left(\mathrm{Z}_{\mathrm{s}}=\mathrm{e}_{3}\right.$ given that $\left.\mathrm{Z}_{\mathrm{t}}=\mathrm{e}_{4}\right)$ for $t, s \in \mathbf{R}$ (Ornstein and Weiss 1991, Werndl 2011). Semi-Markov descriptions are widespread in the sciences (cf. Janssen 1991). 


\section{Observational Equivalence of Deterministic and Indeterministic Descriptions}

Observational equivalence as understood here means that the deterministic description, when the respective system is observed, and the stochastic description give the same predictions. Let me explain what "give the same predictions" means. The predictions derived from a stochastic description are the probability distributions over its realisations. Because there is a probability measure $\mathrm{p}$ defined on a deterministic description, when applying an observation function, the predictions obtained are the probability distributions over the solutions of the description coarse-grained by the observation function. Consequently, a stochastic description $Z_{\mathrm{t}}$ and a deterministic description $\left(X, \mathrm{~T}_{\mathrm{t}} \mathrm{p}\right)$ observed with an observation function $\Phi$ give the same predictions iff: (i) the outcome space $E$ of $\mathrm{Z}_{\mathrm{t}}$ and the set of possible values of $\Phi$ are identical, and (ii) the probability distributions over the solutions of the deterministic description coarse-grained by $\Phi$ and the probability distributions over the realisations are the same.

Suppose that an observation function $\Phi$ is applied to a deterministic description $\left(X, \mathrm{~T}_{\mathrm{t}}, \mathrm{p}\right)$. Then $\left\{\Phi\left(T_{t}\right)\right\}$ is a stochastic description which is observationally equivalent to $\left(X, T_{t}, p\right)$ relative to $\Phi$. Let me explain this with an example. Consider the description of two hard balls in a box (Example 1) and an observation function of this description with four possible values $\mathrm{e}_{1}, \mathrm{e}_{2}, \mathrm{e}_{3}, \mathrm{e}_{4}$. Because a probability measure $\mathrm{p}$ is defined on the phase space $X$, one obtains probabilities such as $\mathrm{P}\left(\Phi\left(\mathrm{T}_{\mathrm{t}}\right)=\mathrm{e}_{1}\right)$ and $\mathrm{P}\left(\Phi\left(\mathrm{T}_{\mathrm{s}}\right)=\mathrm{e}_{2}\right.$ given that $\left.\Phi\left(\mathrm{T}_{\mathrm{t}}\right)=\mathrm{e}_{4}\right)$ for all $t, s \in \mathbf{R}$. Now $\left\{\Phi\left(\mathrm{T}_{\mathrm{t}}\right)\right\}$ is exactly the stochastic description with outcomes $\mathrm{e}_{1}, \mathrm{e}_{2}, \mathrm{e}_{3}, \mathrm{e}_{4}$ and the probability distributions are determined by applying $\Phi$ to the hard ball description. Hence the outcome space of $\left\{\Phi\left(\mathrm{T}_{\mathrm{t}}\right)\right\}$ and the set of possible observed values obtained by applying $\Phi$ to the hard-ball description are identical; and the realisations of $\left\{\Phi\left(\mathrm{T}_{t}\right)\right\}$ and the solutions of the hard ball description coarse-grained by $\Phi$ have the same probability distributions. Thus $\left\{\Phi\left(\mathrm{T}_{\mathrm{t}}\right)\right\}$ and the hard ball description relative to $\Phi$ are observationally equivalent.

The question arises whether the stochastic description $\left\{\Phi\left(\mathrm{T}_{\mathrm{t}}\right)\right\}$ is nontrivial. To highlight the issue: if $\Phi$ is the identity function, $\left\{\Phi\left(\mathrm{T}_{\mathrm{t}}\right)\right\}=\left\{\mathrm{T}_{\mathrm{t}}\right\}$; hence this stochastic description has only trivial probabilities ( 0 and 1$)$ and is really the original deterministic description. It turns out that $\left\{\Phi\left(\mathrm{T}_{\mathrm{t}}\right)\right\}$ is often nontrivial. Let me state a result of Werndl (2011).

Theorem 1. If for the deterministic description $\left(X, \mathrm{~T}_{t}, \mathrm{p}\right)$ there does not exist an $n \in \mathbf{R}^{+}$and a $C \subseteq X, 0<\mathrm{p}(C)<1$, such that $\mathrm{T}_{\mathrm{n}}(C)=C$, then for any arbitrary nontrivial finite-valued observation function $\Phi,\left\{Z_{t}\right\}=\left\{\Phi\left(T_{t}\right)\right\}$ is nontrivial in the following 
sense: for all $t \in \mathbf{R}$, for all $k \in \mathbf{R}^{+}$there are $\mathrm{e}_{\mathrm{i}}, \mathrm{e}_{\mathrm{j}} \in E$ such that $0<\mathrm{P}\left(\mathrm{Z}_{\mathrm{t}+\mathrm{k}}=\mathrm{e}_{\mathrm{i}}\right.$ given that $\left.\mathrm{Z}_{\mathrm{t}}=\mathrm{e}_{\mathrm{j}}\right)<1$.

This result is strong: one obtains nontrivial stochastic descriptions regardless of which finite-valued observation function is applied. Theorem 1 applies to several descriptions in science, e.g., to hard ball descriptions which are important in statistical mechanics; in particular, to descriptions of two hard balls in a box (Example 1) and to almost all descriptions of a finite number of hard balls moving on a torus (Simanyi 1991, Simanyi 2003); to geodesic flows of negative curvature, i.e., frictionless motion of a particle moving with unit speed on a compact manifold with everywhere negative curvature (Ornstein and Weiss 1991); to many billiard descriptions (Chernov 2006); and also to dissipative descriptions such as the Lorenz system which models weather dynamics (Luzzatto 2005).

The discussion so far was about how, given deterministic descriptions, one finds observationally equivalent stochastic descriptions. There are also results about how, given stochastic descriptions, one finds observationally equivalent deterministic descriptions. First, given any stochastic description, one can construct a deterministic description, called the deterministic representation, such that the following holds: the deterministic representation, relative to a specific observation function $\Phi_{0}$, is observationally equivalent to the stochastic description. Yet the phase space of the deterministic representation is defined as consisting of all possible realisations of the stochastic description and thus this construction involves a cheat (Werndl 2011). Apart from the deterministic representation, there are results which show how, given certain kinds of stochastic descriptions, one finds observationally equivalent deterministic descriptions. Let me present two results in this direction.

Theorem 1 tells us that deterministic descriptions in science and stochastic descriptions can be observationally equivalent. Yet it is silent about the nature of these stochastic descriptions. So one might wonder whether deterministic descriptions in science can be observationally equivalent to stochastic descriptions in science (descriptions in science are those that are derived with help of scientific theories). The following theorem shows that the answer is affirmative.

Theorem 2. If the deterministic description $\left(X, \mathrm{~T}_{t}, \mathrm{p}\right)$ is a continuous Bernoulli system, then there are observation functions $\Phi$ such that $\left\{\Phi\left(T_{t}\right)\right\}$ is a semi-Markov description (Ornstein 1970a).

Several deterministic descriptions in science are continuous Bernoulli systems (e.g., all descriptions listed after Theorem 1). Hence several deterministic descriptions in science yield stochastic descriptions in science (semi-Markov descriptions (Example 2)). 
One can go further and ask: can deterministic descriptions in science only yield stochastic descriptions in science for specific observation functions? Or can deterministic descriptions in science yield stochastic descriptions in science regardless at which observation level they are observed? The latter is true.

Theorem 3. If the deterministic description $\left(X, \mathrm{~T}_{\mathrm{t}} \mathrm{p}\right)$ is a continuous Bernoulli system, then for every $\alpha>0,\left(X, \mathrm{~T}_{\mathrm{t}} \mathrm{p}\right)$ is $\alpha$-congruent to a semi-Markov description (Ornstein and Weiss 1991).

Intuitively speaking, being $\alpha$-congruent means to be observationally equivalent at observation level $\alpha$ (cf. Werndl 2011). Several deterministic descriptions in science are continuous Bernoulli systems. Thus Theorem 3 shows that several deterministic descriptions in science are observationally equivalent at every observation level to stochastic descriptions in science (namely semi-Markov descriptions (Example 2)).

Let me now turn to the mathematicians' comments on the results on observational equivalence.

\section{Mathematicians' Comments on Observational Equivalence}

There are hardly any mathematicians commenting on the philosophical significance of the results on observational equivalence. The main exception is the following:

\footnotetext{
Our theorem [Theorem 3] also tells us that certain semi-Markov systems could be thought of as being produced by Newton's laws (billiards seen through a deterministic viewer) or by coin-flipping. This may mean that there is no philosophical distinction between processes governed by roulette wheels and processes governed by Newton's laws. \{The popular literature emphasises the distinction between "deterministic chaos" and "real randomness".\} In this connection we should note that our model for a stationary process $(\S 1.2)$ [the deterministic representation] means that random processes have a deterministic model. This model, however, is abstract, and there is no reason to believe that it can be endowed with any special additional structure. Our point is that we are comparing, in a strong sense, Newton's laws and coin flipping. ${ }^{3}$ (Ornstein and Weiss1991, 39-40)
}

Let me first focus on the claim that there may be no "philosophical distinction between processes governed by roulette wheels and processes governed by Newton's laws". The most direct reading is that there may be no conceptual distinction between deterministic and stochastic descriptions. This seems wrong. This conceptual distinction will always remain, regardless of any results on observational equivalence.

\footnotetext{
${ }^{3}$ The text in braces is in a footnote.
} 
In the above quote Ornstein and Weiss also comment on the meaning of Theorem 3 . On the most plausible reading, they claim that it expresses that deterministic descriptions in science, relative to some observation functions ("viewers"), can be observationally equivalent to stochastic descriptions in science (semi-Markov descriptions). This also illuminates why Ornstein and Weiss mention the deterministic representation, namely, to highlight that this is a case of observational equivalence different from the deterministic representation, which is not a system is science. However, this claim is puzzling. As discussed in the previous section, already Theorem 2 shows that deterministic descriptions in science can be observationally equivalent to semi-Markov descriptions; and Theorem 2 was known before Theorem 3 was proven and is weaker than Theorem 3. Still, this is the most plausible reading. In the previous section I argued that Theorem 3 shows that deterministic descriptions in science are observationally equivalent at every observation level to stochastic descriptions in science. So one expected Ornstein and Weiss to claim this. But this seems not the case because, first, they do not refer to all possible observation levels, and second, if they claimed this, there would be no reason to mention the deterministic representation (cf. Werndl 2011).

Suppes $(1993,254)$ claims that Ornstein and Weiss prove the following (referring to Theorem 3): "There are processes which can equally well be analysed as deterministic systems of classical mechanics or as indeterministic semi-Markov processes, no matter how many observations are made." Clearly, Theorem 3 only proves some results about observational equivalence and not that processes can be equally well analysed as deterministic or indeterministic descriptions. It is not clear that the latter follows from the former. Indeed, I argue in the next section that the results on observational equivalence do not imply that the phenomena can be equally well analysed as deterministic or indeterministic.

Winnie $(1998,310)$ seems to be the only philosopher who explicitly discusses the above quote by Ornstein and Weiss. He takes the claim that there may be no "philosophical distinction between processes governed by roulette wheels and processes governed by Newton's laws" to mean what Suppes (1993) claims, namely the following: the phenomena can be equally well analysed as deterministic or indeterministic descriptions. It is not clear that the absence of the philosophical distinction amounts to the same as that the phenomena can be equally well analysed as deterministic or stochastic; so it is unclear whether Ornstein and Weiss (1991) really want to say this. But if they do, as just mentioned, I will argue in the next section that this is not the case. 


\section{$\underline{\text { V. Winnie on the Role of Different Observations }}$}

\section{V.a Choice and Underdetermination}

We have seen that, in certain cases, deterministic and stochastic descriptions are observationally equivalent. Then there is a choice between a deterministic description and a stochastic description obtained by applying an observation function $\Phi$ to the deterministic description, and the question arises of which description is preferable. I assume that the deterministic and the stochastic description are about the same level of reality, e.g., they both describe the motion of two hard balls. ${ }^{4}$ And I focus on the question of which description is preferable relative to evidence. If none is preferable, there is underdetermination.

It is important to note that there is no underdetermination between a deterministic description and a stochastic description obtained by applying $\Phi$ to a deterministic description relative to all in principle possible observations which show whether there are more states than the ones given by the observation function $\Phi$. In this case only one of the descriptions agrees with the possible observations. This can be seen as follows: suppose that in principle possible observations show that there are more states than the ones given by $\Phi$. Then the stochastic description cannot agree with the observations because it has only the states given by $\Phi$. Conversely, suppose that in principle possible observations show that there are no other states than the ones given by $\Phi$. Then the deterministic description is ruled out because it has more states than the ones given by $\Phi$.

However, other kinds of underdetermination are possible, namely all kinds of underdetermination which are relative to observations which do not show whether there are more states than the ones given by $\Phi$. In particular, suppose that an observation function $\Phi$ is given and that current technology does not allow one to find out whether there are more states then the ones given by $\Phi$ (from the deterministic perspective this means that $\Phi$ is, or is finer than, the finest possible observation function). This implies that the predictions of the deterministic description and of the stochastic description (resulting from applying $\Phi$ ) agree at all currently possible observation levels. If the possible evidence does not favour a description, there is underdetermination relative to all currently possible observa-

\footnotetext{
${ }^{4}$ If the descriptions are about different levels of reality, the situation seems different. For instance, in certain cases one might argue that at one level of reality the deterministic description, and at another level of reality the stochastic description is preferable.
} 
tions (cf. Laudan and Leplin 1991). I take it that Suppes' (1993) claim that phenomena are equally well analysable by deterministic or stochastic descriptions implies that there is underdetermination.

In what follows I concentrate on an argument against underdetermination by Winnie (1998). I will criticize this argument and, in particular, I will clarify a confusion in it.

\section{V.b Trivial Transition Probabilities to Coarser Observations}

Winnie (1998) starts with the following thought. For a deterministic description $\left(X, \mathrm{~T}_{\mathrm{t}} \mathrm{p}\right)$ consider observation functions $\Psi$ and $\Phi$ such that $\Phi$ is coarser than $\Psi$, i.e., there is at least one value of $\Phi$ such that two or more values of $\Psi$ correspond to one value of $\Phi$, and a value of $\Phi$ always corresponds to one or more values of $\Psi$. Even if $\left\{\Phi\left(\mathrm{T}_{\mathrm{t}}\right)\right\}$ and $\left\{\Psi\left(\mathrm{T}_{\mathrm{t}}\right)\right\}$ are nontrivial stochastic descriptions, the following can hold for a time period $t$ : for every value $\mathrm{O}_{\Psi}$ of $\Psi$ and every value $\mathrm{o}_{\Phi}$ of $\Phi$ the probability that $\mathrm{O}_{\Psi}$ will lead to $\mathrm{O}_{\Phi}$ after $t$ time steps is 0 or 1 . Thus there are trivial transition probabilities from the observation modeled by $\Psi$ to the coarser observation modeled by $\Phi$, where the transition probabilities are the probabilities that any arbitrary value follows another arbitrary value. ${ }^{5}$ Winnie $(1998,314-315)$ comments on this:

Thus, the fact that a chaotic deterministic system [...] has some partitioning that yields a set of random or stochastic observations in no way undermines the distinction between deterministic and stochastic behaviour for such systems. [...] As successive partitionings are exemplified [...] the determinism underlying the preceding, coarser observations emerges. To be sure, at any state of the above process, the system may be modeled stochastically, but the successive stages of that modeling process provide ample inductive - reason for believing that the deterministic model is correct [original emphasis].

${ }^{5}$ To give an example, on $X=[0,1] \mathrm{x}[0,1]$ consider:

$\mathrm{T}((x, y))=(2 x, y \backslash 2)$ if $0 \leq x<1 \backslash 2,(2 x-1,(y+1) / 2)$ if $1 \backslash 2 \leq x \leq 1$.

For the Lebesgue probability measure $\mathrm{p}$ one obtains the discrete-time deterministic description $\left(X, \mathrm{~T}_{\mathrm{t}} \mathrm{p}\right)$, called the baker's transformation. Consider $\Phi((x, y))=\mathrm{o}_{1} \chi_{\alpha 1}((x, y))+\mathrm{o}_{2} \chi_{\alpha 2}((x, y))$, where $\alpha 1=[0,1] \mathrm{x}[0,1 / 2], \alpha 2=[0,1] \mathrm{x}(1 / 2,1]$ and $\Psi((x, y))=\mathrm{q}_{1} \chi_{\beta 1}((x, y))+\mathrm{q}_{2} \chi_{\beta 2}((x, y))+\mathrm{q}_{3} \chi_{\beta 3}((x, y))+\mathrm{q}_{4} \chi_{\beta 4}((x, y))$, where $\beta 1=[0,1 / 2] \times[0,1 / 2], \quad \beta 2=(1 / 2,1] \times[0,1 / 2], \quad \beta 3=[0,1 / 2] \times(1 / 2,1], \beta 4=(1 / 2,1] \times(1 / 2,1]$ $\left(\chi_{A}(z):=1\right.$ for $z \in A ; 0$ otherwise). Clearly, if one observes $q_{1}$ (with $\left.\Psi\right)$, the probability that one next observes $o_{1}$ (with $\Phi$ ) is 1 ; if one observes $q_{2}$, the probability that one next observes $\mathrm{o}_{2}$ is 1 ; if one observes $\mathrm{q}_{3}$, the probability that one next observes $\mathrm{o}_{1}$ is 1 ; and if one observes $\mathrm{q}_{4}$, the probability that one next observes $\mathrm{o}_{2}$ is 1 . 
In order to understand this quote, note the following. From the fact that there are trivial transition probabilities from an observation $(\Psi)$ to a coarser observation $(\Phi)$ after $t$ time steps, it does not follow that the observed phenomenon is deterministic and Winnie also does not claim this. It may be that $\left\{\Psi\left(\mathrm{T}_{\mathrm{t}}\right)\right\}$, or any stochastic description at a smaller scale, is the correct description.

The argument Winnie (1998) seems to make is the following. Relative to the notion of underdetermination of interest, consider the observation functions which, according to the deterministic description, one should be able to apply (i.e., corresponding to observations which, according to the deterministic description, are in principle possible or possible given current technology). Suppose that the observations corresponding to these observation functions can be made (i.e. are in principle possible or possible given current technology). Suppose that for some observation functions there are trivial transition probabilities from finer to coarser observation functions after $t$ time steps for some fixed time $t$. Now consider all observation functions such that there are trivial transition probabilities from finer to coarser observation functions, and suppose that one finds that the application of finer observation functions leads to stochastic descriptions at a smaller scale (i.e., descriptions where there is at least one outcome of the stochastic description at a larger scale such that two or more outcomes of the stochastic description at a smaller scale correspond to one outcome of the description at a larger scale; and an outcome of the description at a larger scale always corresponds to one or more outcomes of the description at a smaller scale). Then the deterministic description is preferable relative to evidence.

My first criticism is that it is unclear why this argument requires that there are trivial transition probabilities from finer to coarser observation functions. The force of the argument seems only that finer observations can be made, that for finer observation functions one obtains stochastic descriptions at a smaller scale, and that the stochastic descriptions at a smaller scale explain how the probabilities of the stochastic description at the larger scale arise. Simple examples show that there are observation functions such that finer observation functions yield stochastic descriptions at a smaller scale, but the transition probabilities are not always (or even never) trivial. ${ }^{6}$ The force of the argument also seems to apply to these examples. From the text it is not entirely clear whether Winnie thought that trivial transition probabilities to coarser observations are decisive for the argument that the deterministic description is preferable. If yes, as just argued, this is puzzling be-

6 For example, consider the baker's transformation $\left(X, \mathrm{~T}_{\mathrm{t}} \mathrm{p}\right)$. Let $\Psi((x, y))=\mathrm{q}_{1} \chi_{\beta 1}((x, y))+\mathrm{q}_{2} \chi_{\beta 2}((x, y))+\mathrm{q}_{3} \chi_{\beta 3}((x, y))+\mathrm{q}_{4} \chi_{\beta 4}((x, y))$ be as in the previous footnote and let $\Phi((x, y))=\mathrm{o}_{1} \chi_{\gamma 1}((x, y))+\mathrm{o}_{2} \chi_{\gamma 2}((x, y)), \quad \gamma 1=[0,1 / 2] \mathrm{x}[0,1]$, $\gamma_{2}=(1 / 2,1] x[0,1]$. Clearly, for all $\mathrm{i}, 1 \leq \mathrm{i} \leq 4$, and all $\mathrm{j}, 1 \leq \mathrm{j} \leq 2$, the probability that $\mathrm{q}_{\mathrm{i}}$ is followed by $o_{j} 1 / 2$. Still $\Phi$ is coarser than $\Psi$, and for the observation $\Psi$ at the finer level one obtains a stochastic description at a smaller scale. 
cause the force of the argument does not seem to hinge on this. If not, it is confusing that trivial transition probabilities to coarser observations are highlighted in the way they are.

I will now criticize Winnie's argument; my criticism applies regardless of whether one requires that the observation functions are such that trivial transition probabilities are observed from finer to coarser observations.

\section{$\underline{\text { V.c Criticism of Winnie's Argument About Finer Stochastic }}$ Processes}

Winnie does not state which kind of underdetermination he is concerned with. Suppose that it is underdetermination relative to in principle possible observations which show whether there are more states than the ones given by $\Phi$. As argued at the beginning of this section, here it is easy to see that there is no underdetermination. Then Winnie's argument indeed delivers the correct conclusion: the deterministic description is preferable just in case there are more states than the ones given by $\Phi$. And there are more states than the ones given by $\Phi$ just in case the in principle possible observations (according to the deterministic description) can be made; and then it automatically follows that finer observation functions lead to stochastic descriptions at a smaller scale. Still, Winnie's argument seems complicated. The reason why there is no underdetermination is simply that only the deterministic description agrees with the in principle possible observations.

Suppose that Winnie is concerned with underdetermination relative to all possible observations given current technology. Then, I will argue, Winnie's argument fails. To show this, it suffices to present a scenario that could happen in science (regardless of whether this is actually the case) where the premises are true but the conclusion is not. Let me outline such a scenario.

This scenario appeals to indirect evidence, which is generally regarded as an important kind of evidence (Laudan 1996, Laudan and Leplin 1991, Okasha 2002). Let me given an example of indirect evidence (cf. Laudan 1996). Darwin's hypothesis of natural selection is only about selection which is natural and not artificial. Despite this, data from breeders about artificial selection provide evidence for Darwin's theory about natural selection. Data from breeders support Darwin's general theory of evolution and so provide indirect evidence for the theory of natural selection (even though these data are not derivable from the theory of natural selection). As Laudan and Leplin (1991) point out, indirect evidence can be an argument against underdetermination. Suppose that the same predictions are derivable from a hypothesis $H$ than from the theory of natural selection but that $H$ is not derivable from Darwin's general theory of evolution. Because of the indirect evi- 
dence for the theory of natural selection, it is preferable to $H$ relative to evidence, and there is no underdetermination.

An analogous argument for our concern - descriptions and not hypotheses - can easily be found. Suppose that the stochastic description $S$ which arises from applying $\Phi$ to the deterministic description derives from a well-confirmed theory $W$ and the deterministic description $D$ does not derive from any theory. Furthermore, suppose that current technology does not allow one to find out whether there are more states than the ones given by $\Phi$. This means that regardless of whether the stochastic or deterministic description is correct, one can make the observations corresponding to the observation functions one is interested in. Furthermore, for the observation functions which one can apply (and where, maybe, only those possible observation functions are considered where there are trivial transition probabilities from finer to coarser observations) the following holds: for finer observation functions one obtains stochastic descriptions at a smaller scale. Hence the premises of Winnie's argument are true. Now even though $S$ and $D$ are observationally equivalent, there are many descriptions which are not derivable from $S$ or $D$ but which support $W$. Suppose that some of these descriptions provide indirect evidence for $S$. Then the stochastic description $S$ is preferable relative to evidence, and the conclusion of Winnie's argument is not true. Also, there is no underdetermination; consequently, Suppes' (1993) claim that the phenomena are equally well analysable as deterministic or stochastic descriptions fails.

What does indirect evidence amount to? Note that being derivable from the same hypothesis or statement cannot be sufficient for indirect evidence because this would lead to the paradox that any statement confirms any statement. ${ }^{7}$ A promising account is that there is indirect confirmation when two statements are strongly coherent because of a unifying theory; in our examples this coherence is provided by Darwin's general theory about evolution, by Newtonian theory and by the theory $W$.

\section{Conclusion}

This paper started by presenting some results on the observational equivalence of deterministic and indeterministic descriptions. Then I examined philosophical questions prompted by these results.

\footnotetext{
${ }^{7}$ Statement $A$ confirms itself; $A$ is derivable from $A \& B$ (B is any statement); $B$ is derivable from $A \& B$. Hence, $A$ confirms $B$.
} 
First, I discussed the philosophical comments made by mathematicians about observational equivalence, namely Ornstein and Weiss (1991), and I argued that they are misguided. For instance, on a direct reading, Ornstein and Weiss claim that the results on observational equivalence may show that there is no conceptual distinction between deterministic and indeterministic descriptions. However, regardless of any results on observational equivalence, this distinction remains.

Second, if there is a choice between a deterministic and an indeterministic description, the question arises of which description is preferable. I investigated Winnie's (1998) argument that the deterministic description is preferable which goes as follows. Consider the possible observation functions which, according to the deterministic description, one should be able to apply. Suppose that these observations can be made and that there are trivial transition probabilities from finer to coarser observations after $t$ time steps. Further, suppose that stochastic descriptions at a smaller scale are obtained when finer observation functions are applied. Then the deterministic description is preferable relative to all evidence. I clarified a confusion in this argument: it unclear why trivial transition probabilities are required

from finer to coarser observations because the force of the argument does not seem to hinge on this. Then I argued that, regardless of this, if the concern is a strong form of underdetermination, the argument delivers the desired conclusion but this conclusion follows from a much simpler argument. And if the concern is underdetermination relative to the possible observations given current technology, the argument fails. The question of whether the deterministic or the stochastic description is preferable is an interesting one and, as my discussion has hopefully shown, it deserves further investigation.

Acknowledgements

I am indebted to Jeremy Butterfield for valuable comments. For stimulating discussions I am grateful to Franz Huber, James Ladyman, Miklos Redei, Jos Uffink, and the audiences at the Oxford Philosophy of Physics Research Seminar, the Bristol Philosophy of Science Research Seminar, and the EPSA conference 2009. This research has been supported by a Junior Research Fellowship from the Queen's College, Oxford University.

\section{$\underline{\text { References }}$}

Chernov, Nikolai and Roberto Markarian. 2006. Chaotic billiards. Providence: American Mathematical Society.

Janssen, Jacques and Nikolaos Limnios. 1999. Semi-Markov models and applications, Dordrecht: Kluwer Academic Publishers.

Laudan, Larry. 1995, Damn the consequences! Proceedings and Addresses of the American Philosophical Association 69:27-34. 
Laudan, Larry and Jarrett Leplin 1991. Empirical equivalence and underdetermination. The Journal of Philosophy 88:449-472.

Luzzatto, Stefano, Melbourne, Ian and Frederic Paccaut. 2005. The Lorenz attractor is mixing. Communications in Mathematical Physics 260:393-401.

Okasha, Samir. 2002. Underdetermination, holism and the theory/data distinction. The Philosophical Quarterly 208:303-319.

Ornstein, D. 1970. Imbedding Bernoulli shifts in flows. In Contributions to ergodic theory and probability, eds. Albrecht Dold and Beno Eckmann, 178-218. Berlin: Springer.

Ornstein, Dan and Benjamin Weiss. 1991. Statistical properties of chaotic systems. Bulletin of the American Mathematical Society 24:11-116.

Simanyi, Nandor. 1999. Ergodicity of hard spheres in a box. Ergodic Theory and Dynamical Systems 19:741-766.

Simanyi, Nandor. 2003. Proof of the Boltzmann-Sinai ergodic hypothesis for typical hard disksystems. Inventiones Mathematicae 154:123-178.

Suppes, Patrick. 1993. The transcendental character of determinism. Midwest Studies in Philosophy 18:242-257.

Werndl, Charlotte. 2009a. Are deterministic descriptions and indeterministic descriptions observationally equivalent? Studies in History and Philosophy of Modern Physics 40:232-242.

Werndl, Charlotte. 2009b. What are the new implications of chaos for unpredictability? The British Journal for the Philosophy of Science 60:195-220.

Werndl, Charlotte. 2011. On the observational equivalence of continuous-time deterministic and indeterministic descriptions. European Journal for the Philosophy of Science. doi: 10.1007/S1319401000115.

Winnie, J. 1998. Deterministic chaos and the nature of chance. In The cosmos of science - essays of exploration, eds. John Earman and John Norton, 299-324. Pittsburgh: Pittsburgh University Press. 